\title{
The use of indocyanine green imaging technique in patient with hepatocellular carcinoma
}

\author{
Shih-Wei Huang ${ }^{1,2 \#}$, Jing-Jim Ou ${ }^{3 \#}$, Hon Phin Wong ${ }^{1,2}$ \\ ${ }^{1}$ Division of General Surgery, Department of Surgery, Show Chwan Memorial Hospital, Changhua, Taiwan; ${ }^{2}$ IRCAD/AITS-Asian Institute of \\ TeleSurgery, Show Chwan Health Care System, Changhua, Taiwan; ${ }^{3}$ Department of Surgery, Chang Bing Show Chwan Memorial Hospital, Lukang \\ Town, Changhua, Taiwan \\ Contributions: (I) Conception and design: All authors; (II) Administrative support: All authors; (III) provision of study materials or patients: All \\ authors; (IV) collection and assembly of data: All authors; (V) data analysis and interpretation: All authors; (VI) manuscript writing: All authors; \\ (VII) final approval of manuscript: All authors. \\ "These authors contributed equally to this work. \\ Correspondence to: Hon Phin Wong. Division of General Surgery, Department of Surgery, Show Chwan Memorial Hospital, No. 542, Chung-Shan \\ Rd. Sec. 1, Changhua 500, Taiwan. Email: wdolphin790622@gmail.com.
}

\begin{abstract}
Near-infrared indocyanine green (ICG) fluorescence application in liver cancer surgery have been reported in the literature since 2008. To date, most reports emphasized not only to the safety, feasibility and reproducibility, but also the potential benefits of its clinical applications in term of demarcating segmentation for an anatomical resection, tumor identification to achieve tumor free resection margin, detection of small unidentifiable subcapsular nodules as well as extrahepatic metastatic lesions, and fluorescence cholangiography. The purpose of this review is to present the fundamental concept of the interpretation of fluorescence enhancement by different timing through intravascular ICG distribution to liver and biliary washout; to describe step-by-step technical aspects of its use in different purposes, and to expose the diagnostic and therapeutic perspectives of this innovative imaging technique in liver cancer surgery.
\end{abstract}

Keywords: Hepatocellular carcinoma (HCC); indocyanine green; near-infrared

Received: 29 September 2018; Accepted: 29 October 2018. Published: 21 November 2018.

doi: $10.21037 / \operatorname{tgh} .2018 .10 .15$

View this article at: http://dx.doi.org/10.21037/tgh.2018.10.15

\section{Introduction}

Clinically, indocyanine green (ICG) retention rate at 15 minutes (ICG R15) is the most common perioperative dynamic assessment of liver function that indicates the upper limit of the hepatectomy procedure in patients with hepatocellular carcinoma (HCC) and liver cirrhosis (1). After being approved by FDA in 1950s, ICG was initially used primarily in hepatic function diagnostics (2). Its fluorescence property in which protein-bound ICG emits fluorescence that peaks at about $840 \mathrm{~nm}$ when illuminated with near-infrared (NIR) light was then proposed in detail in the 1970s (3). Moreover, the first application of this imaging technique in hepatic surgery was reported by Aoki et al. (4) in 2008 to identify segment and subsegment for anatomical hepatic resection and Ishizawa et al. in 2009 for tumor identification. ICG fluorescence imaging in abdominal surgery was initially limited to open procedures. As laparoscopic fluorescence imaging systems were developed for clinical applications after 2010, ICG fluorescence imaging was widely applied in minimally invasive abdominal surgery, especially for visualizing extrahepatic bile ducts during laparoscopic/robotic cholecystectomy (5), which is known as fluorescence cholangiography (6) and can also be applied in liver cancer surgery.

In 2016 Asia Pacific consensus statement on laparoscopic liver resection held in Hong Kong (7), ICG fluorescence imaging was highlighted with the advantages of real time 


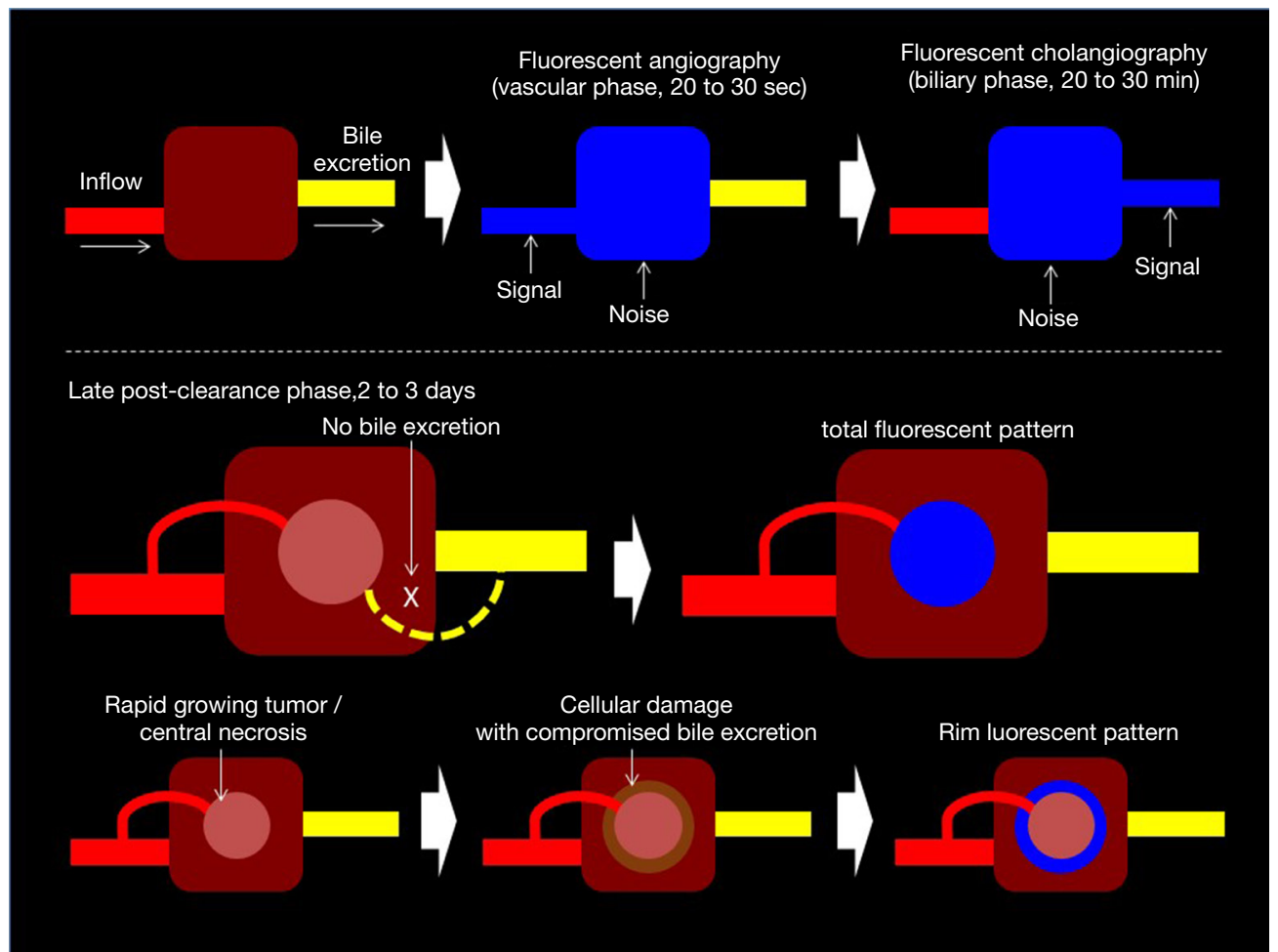

Figure 1 During ICG IV administration, tissue concentration contrast and hence fluorescence contrast is determined by intravascular contributions (vascular phase, about 20 to 30 seconds after administration). As ICG is cleared by the liver and excreted into the bile ducts, an interval of at least 20 to 30 minutes after administration was needed until the fluorescence cholangiography can be identified (biliary phase), while the liver target may remain obscured (noise-to-signal ratio) for 2 to 3 days depends on the liver function. After adequate clearance time, contrast between the target and surrounding tissue is achieved (late post-clearance phase). ICG, indocyanine green.

illumination of occult lesions $(8,9)$ showing a more precise pathology in surgical specimens $(10,11)$. Intraoperative applicability has been described in revealing the surgical margins (12) and identifying anatomical landmarks of the liver $(4,13,14)$. This tool has received acceptance in various surgical disciplines, and it may become standard equipment in near future. However, the technology is relatively new, it may be difficult to demonstrate a statistically significant clinical difference with its application.

This review focuses on mainly the different clinical applications in liver cancer surgery with an emphasis on diagnostic and therapeutic prospective the near-infrared fluorescence (NIRF) can achieve; moreover, practical information on doses, injection times, and intraoperative use are provided.

\section{Materials and methods}

We searched for original articles focusing on NIRF imaging to HCC in PubMed published between 2008 and 2018. The search terms we used were 'indocyanine green', 'fluorescence', 'liver neoplasms'. All papers identified were English full-text papers limited to human. We also searched the reference lists of identified articles for further papers. Articles focusing on liver neoplasm other than HCC, case reports, and animal studies were excluded.

\section{Concept of ICG NIRF application in HCC}

ICG fluorescent signal is more prominent after binding to plasma protein such as albumin, lipoprotein and ligandin in vivo (15-17). It was proposed that HCC cells can take up ICG like normal hepatocytes; however, ICG secretion from cancer cells into the bile is impaired $(10,18)$ (Figure 1). Ligandin (glutathione S-transferases) is uniformly distributed over hepatocytes in a normal liver. The expression of ligandin decreases with liver damage, and it is not expressed in areas of necrosis, fibrosis, or 


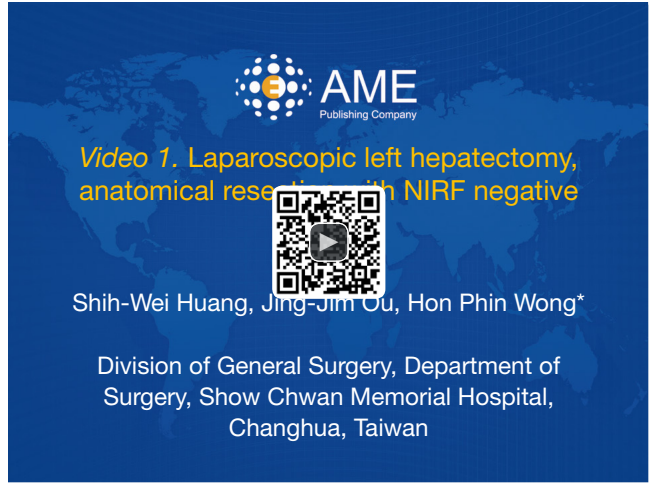

Figure 2 Laparoscopic left hepatectomy, anatomical resection with NIRF negative contrast. A step-by-step demonstration through hilar approach. The parenchymal transection demarcation was clearer under NIRF view that even the bare area covered with coronary ligament could be clearly seen due to the penetrating property of near infrared wave that can go through a certain depth of soft tissue. Fluorescence cholangiography to identify the main bile duct confluence provide targeted signal for dissection and ligation that may avoid the risk of stricture (24). NIRF, near-infrared fluorescence.

Available online: http://www.asvide.com/article/view/28308

severe inflammation, thus the expression of ligandin becomes relatively rich in the regenerative area (19). Furthermore, the gene and protein expression levels for $\mathrm{Na}^{+}$/taurocholate cotransporting polypeptide (NTCP) and organic anion transporting polypeptide 8 (OATP8), which are associated with portal uptake of ICG by hepatocytes were demonstrated to be higher in the HCCs that showed cancerous-type fluorescence than in those that showed rim-type fluorescence, showing fluorescence only in the surrounding non-cancerous liver parenchyma. Therefore, preserved portal uptake of ICG in differentiated HCC cells by NTCP and OATP8 with concomitant biliary excretion impairment causes accumulation of ICG in the cancerous tissues after preoperative intravenous administration. This allows highly sensitive visualization of the pharmacokinetics of organic anions such as ICG in HCC tissues by intraoperative ICG fluorescence imaging (20).

\section{Clinical application in HCC}

\section{Segmentation mapping: positive- and negative-staining techniques [intravenous (IV) and portal vein (PV) approaches]}

Since anatomical resection of the liver was first reported by Makuuchi et al. in 1985 (21), it has played an important role in the treatment of hepatic malignancies; however, surgeons may encounter difficulties in harvesting clear demarcation of the hepatic segments based on nakedeye before parenchymal transection, which in turn, may decrease the accuracy of anatomical segmentectomy. Takasaki et al. proposed a similar concept in term of anatomical resection that the Glissonean pedicles can be transected intrahepatically or extrahepatically to identify the boundaries of the intersegmental planes (22).

\section{PV approach}

Based on the same principle as dye tattooing of the liver segment to be resected by injecting ICG into the portal vein under intraoperative ultrasound (US) guidance (21), intraoperative fluorescence allows positive enhancement of the segments to be resected, in order to perform a formal liver resection (4). The handling of ICG injection speed under US guidance into the portal vein is relatively technically demanding, as there is a chance of regurgitation or spillover into the noneligible branches. Sakoda et al. reported a puncture method for the target portal branch under percutaneous US with artificial ascites in the case of laparoscopic hepatectomy, which further emphasize the technical difficulty to determine the portal vein territory by staining under intraoperative laparoscopic and percutaneous US (23).

\section{IV approach}

Through the Takasaki hilar approach of anatomical resection, the associated Glissonean pedicle transection can then clearly identifying the boundaries of the intersegmental planes (22). Through this approach (Figure 2), a negative contrast delineation can be achieved via an easier IV ICG injection. The surgical difficulty to this technique is one must be familiar to the cone unit principle with the understanding of the possible variations intrahepatic secondary and tertiary Glissonean pedicles (25).

\section{Identification of liver lesions}

ICG, a non-specific molecule, can be uptaked by differentiated HCC cells and remained in the cytoplasm and/or pseudo glands for several weeks after intravenous injection, which allows identification of tumoral tissues by intraoperative ICG fluorescence imaging with high sensitivity (20). Late post-clearance fluorescence illumination observed several days after ICG had been 
largely washed out from blood by liver, and showed targeted lesion enhancement at high contrast over a rather dim homogeneous normal liver parenchymal background (Figure 1). This can achieve the goals to detect multifocal small lesions which are unidentified on gross white light atmosphere (12). Moreover, the tumor fluorescence enhancement can provide real-time demarcation of liver tumor which is beneficial to harvest a tumor free cut margin especially in non-anatomical resection.

The summary of the ICG applications are shown in Table 1, and their advantages are delineated in the following. Most publications are from eastern countries, predominantly Japan. The dose of ICG most frequently used was 1.25 to $5 \mathrm{mg}$ IV or PV intraoperatively to identify hepatic segment; and, $0.5 \mathrm{mg}$ per $\mathrm{kg}$ of body weight administrated, as ICG R15 test, several days prior to surgery for liver tumors identification. The interval of ICG application before surgery was ranged from few minutes to 28 days.

\section{Identifying bepatic segment and subsegment}

In 2008, Aoki et al. (4) reported an intraoperative technique for identifying segment and subsegment of the liver with high-sensitivity NIRF imaging for anatomical hepatic resection. Stained subsegments and segments of the liver were identifiable in $33(94.3 \%)$ of the 35 patients. Fluorescence segmentation was concluded safe and reproducible. Their following study in 2010 further demonstrated that this method for clear mapping of liver segments even against a background of cirrhosis. Moreover, they also introduced NIRF cholangiography during laparoscopic cholecystectomy (26).

Miyata et al. (33) further reported the concomitant use of ICG fluorescence imaging with indigo-carmine staining is feasible and may enhance the detectability of hepatic segments during anatomical resection, especially in those whose liver is covered with connective tissues from previous surgery. ICG fluorescence imaging is also potentially useful in patients with liver cirrhosis because the irregularity of the liver surface and fibrosis of the hepatic parenchyma can decrease contrast between indigo-carmine-stained hepatic parenchyma and unstained regions of the liver, although this could not be confirmed statistically in this study.

Alternatively, Uchiyama et al. (27) advocated combining fluorescence navigation system (PDE) using ICG and contrast-enhanced intra-operative US with Sonazoid for detection of liver sections and segments, and demonstrated it to be a useful and safe tool for performing liver resection.
It was proved that the resection line using PDE was clearly detected in all 22 patients $(\mathrm{P}<0.018)$.

Through laparoscopic setting, Sakoda et al. (30) identified a portal vein territory feeding the domain by ICG injection for NIRF imaging under the guidance of laparoscopic US in order to achieve pure laparoscopic anatomical resection for small HCC (Table 1). This method is considered to be safe with both low invasiveness and curative success. Kobayashi et al. (37) further demonstrated the technical details of five types of fluorescence staining techniques including single staining, multiple staining, counterstaining, negative staining and paradoxical negative staining. These techniques were proven to be safe and facilitate accurate visualization of the PV territory in real time, improving the efficacy of anatomical removal of PV territories.

Inoue et al. (14) reported their applications of fusion ICG-fluorescence imaging in which pseudocolorfluorescence images are superimposed on white-light color images in real time to the three-dimensional identification of liver territories. By comparing with conventional demarcation technique, fusion ICG-fluorescence imaging obtained more precise and clearer demarcation for anatomical liver resection.

The recent study of Terasawa et al. (35) demonstrated that fusion ICG-fluorescence imaging enhances its feasibility in identifying of hepatic tumors and segmental boundaries during laparoscopic hepatectomy (Table 1). Thus, it might assist surgeons complete laparoscopic hepatectomies safely and accurately. Also, Boogerd et al. (36) proved the added benefit of fluorescence imaging in laparoscopic resections for several hepatic tumors by demarcating liver tumors and thus providing real-time resection margin assessment, and identifying otherwise undetectable occult liver tumors.

\section{Identification of liver cancers}

\section{Identification of subcapsular liver cancers}

In 2009, Ishizawa et al. reported the first NIR/ICG fluorescence application in liver tumor identification. Eight of 63 HCCs were identified with NIRF imaging that were otherwise imperceptible; $8 \%$ of false-positives rate was reported (10). By 2013, the series progressed to 170 subjects and 276 HCCs. False-positive rate dropped to $1 \%$, while 273 of 276 lesions (99\%) were identified under NIRF, including 21 grossly unidentifiable lesions (20).

Morita et al. (29) further evaluated the ICG fluorescence imagings and showed that ICG fluorography identified 


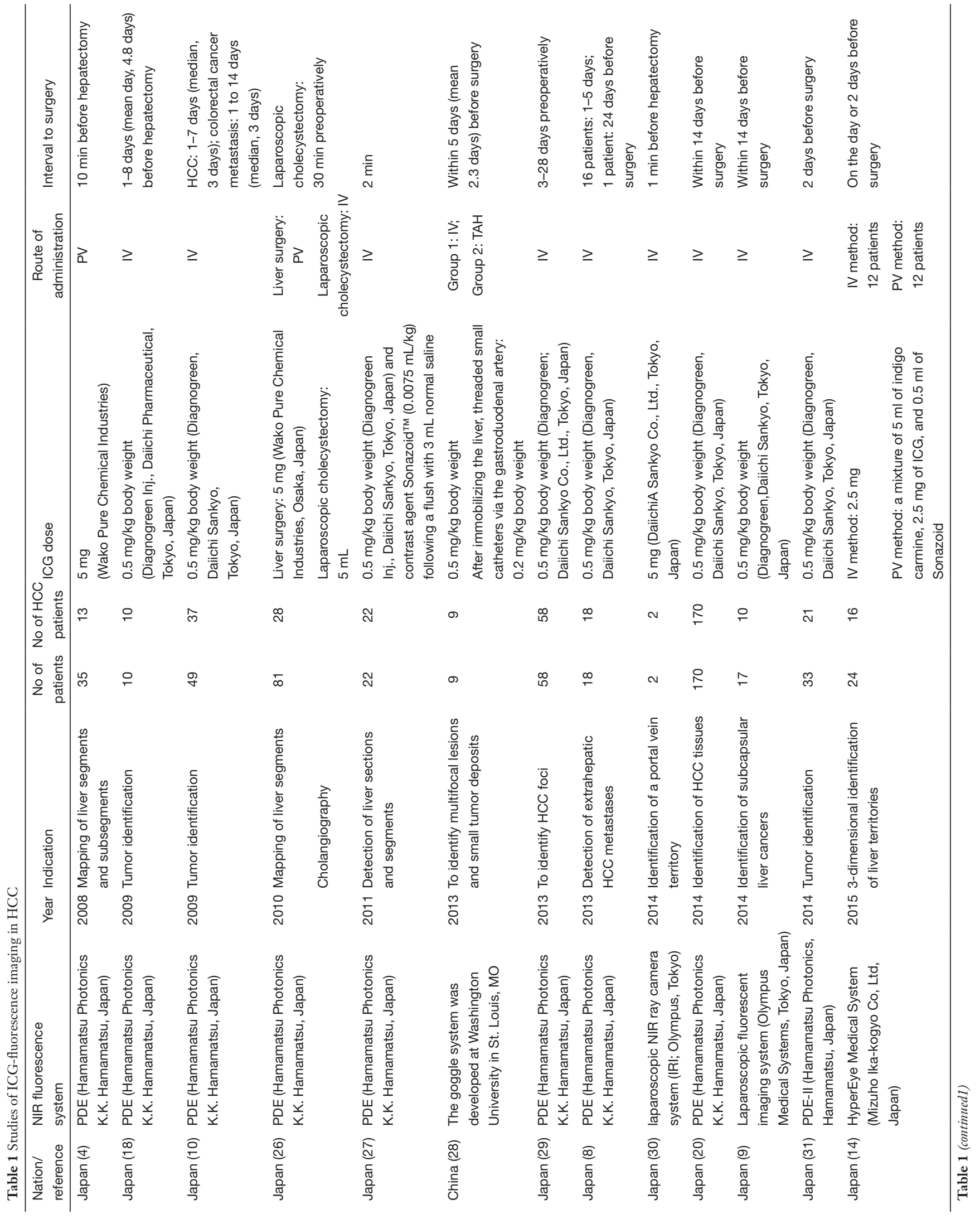




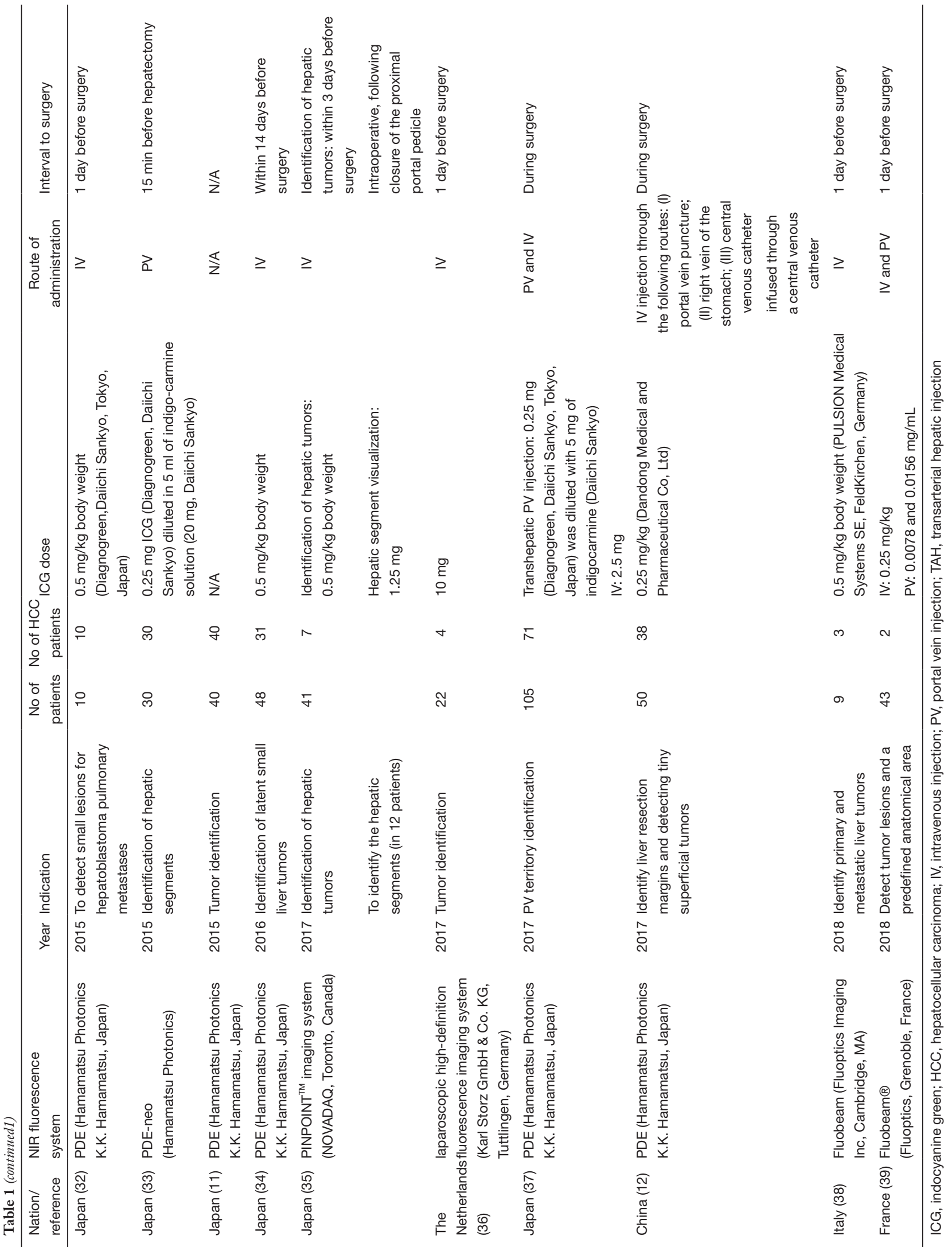


73 of $76(96 \%)$ preoperatively diagnosed HCC lesions. Other than preoperatively diagnosed foci, NIRF visualized 35 new lesions, including 6 HCCs, 2 dysplastic nodules and 27 non-neoplastic lesions, such as bile plugs and cysts. Overall, the sensitivity of NIRF for HCCs was $96 \%$ and its positive predictive value was $71.5 \%$. As a result, Morita et al. also proved NIRF is useful for detecting small liver tumors, especially for HCC; however, the same issue was discussed in doubt of its sensitivity as some lesions visualized under NIR view were not neoplastic.

Tanaka et al. (31) got the same results as Ishizawa's report concerning with illuminated pattern of liver tumors (10). It was clear that although false positive nodules were not observed in the non-cirrhotic livers, smaller tumors and tumors that were located deeper than $10 \mathrm{~mm}$ from liver surface to tumor were difficult to recognize as illuminated nodules. Presumably, $10 \mathrm{~mm}$ is the maximum limit of depth for ICG-FNS to detect tumors (31).

Kudo et al. (9) devised the technique for laparoscopic ICG fluorescence imaging and evaluated the efficacy for identifying subcapsular liver cancers in laparoscopic hepatectomy. It was described that like palpation during open hepatectomy, this technique enables real-time identification of subcapsular liver cancers, subsequently facilitating estimation of the required extent of hepatic mobilization and determining the location of an appropriate hepatic transection line.

Kaibori et al. (34) compared the sensitivity, specificity and accuracy of ICG and 5-ALA fluorescence imaging in detecting tumors. The sensitivity, specificity and accuracy of ICG in detecting the carcinomatous main tumors were 96\% (44/46), 50\% (1/2) and 94\% (45/48), respectively. There were nine latent small tumors newly detected on the liver surface using ICG fluorescence imaging, of which five were carcinomas. The sensitivity, specificity and accuracy of 5-ALA for detecting the carcinomatous main tumors were $57 \%(26 / 46), 100 \%(2 / 2)$ and $58 \%$ (28/48), respectively. Among the five latent small tumors newly detected on the liver surface by 5 -ALA fluorescence imaging, all were identified as carcinomas. Thus, sensitivity and specificity of ICG fluorescence imaging for main tumor detection were relatively high and low, respectively, but the opposite was for 5-ALA imaging. Therefore, 5-ALA may provide greater specificity in the detection of surface-invisible liver tumors than using ICG fluorescence imaging alone. The main advantages of ICG are its safety and its commercial availability as a contrast agent (18).

\section{Identifying multifocal lesions and small tumor deposits}

Recently, Zhang et al. (12) demonstrated that intraoperative ICG fluorescence navigation system revealed 12 small tumors in 8 patients in whom a preoperative imaging examination did not indicate the existence of these tumors. The smallest lesion was approximately $2 \mathrm{~mm}$ in diameter. Therefore, intraoperative ICG fluorescence imaging navigation enabled the high sensitivity for identifying tiny and grossly unidentifiable liver cancer tumors in real time, enhancing the accuracy of liver resection and operative cancer staging.

\section{Identification of metastasis}

Identifying extrahepatic metastatic lesions from HCC In the advanced stage of HCC, extrahepatic metastases often occur in lung, lymph node, adrenal gland, peritoneum, and elsewhere. The first report showing that extrahepatic metastases from HCC retained ICG after intravenous injection, and exhibited fluorescence when illuminated with near-infrared light, indicating their capability to transport ICG, was conducted by Satou et al. (8). This technique can be a useful tool for intraoperative identification of extrahepatic metastatic lesions in HCC patients.

Also, the recent study by Lieto et al. (38) proved that ICG fluorescence imaging accurately identified primary and metastatic liver tumors. Besides, ICG fluorescence imaging was capable to detect small, superficial and subcapsular tumor nodules that have been unrecognized by other diagnostic tools. Therefore, ICG fluorescence imaging is an effective tool to improve both intraoperative staging and radicality in the surgery of primary and metastatic liver tumors.

\section{Detection of small pulmonary metastases}

It was reported that ICG techniques was very useful for the detection of small pulmonary metastases because of the high tissue contrast, though the problem of false positive remains (32). 


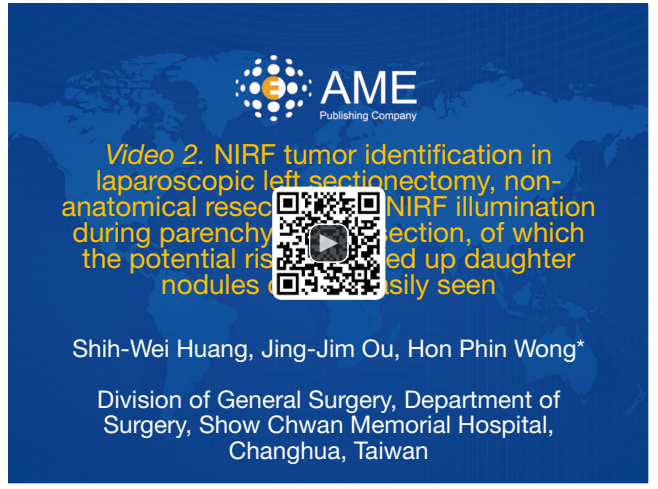

Figure 3 NIRF tumor identification in laparoscopic left sectionectomy, non-anatomical resection with NIRF illumination during parenchymal transection, of which the potential risk of missed up daughter nodules can be easily seen (43). NIRF, near-infrared fluorescence.

Available online: http://www.asvide.com/article/view/28309

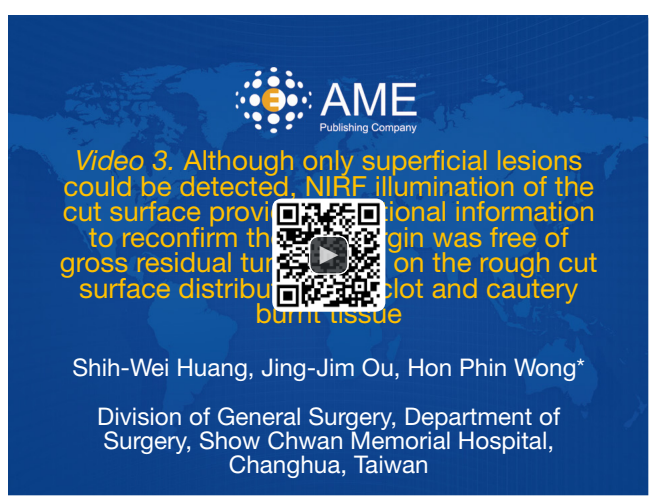

Figure 4 Although only superficial lesions could be detected, NIRF illumination of the cut surface provided additional information to reconfirm the cut margin was free of gross residual tumor, even on the rough cut surface distributed with clot and cautery burnt tissue (44). NIRF, near-infrared fluorescence.

Available online: http://www.asvide.com/article/view/28310

\section{Discussion}

The time between ICG injection and hepatectomy of 1 to 28 days has also been the topic of several publications $(10,18$, 29,31,32,36,38,39), while interval not more than 3 days (35) and 2 weeks $(20,28,34,40)$ were also proposed by other groups. The interval is related to liver function: poor liver function and cirrhosis (41) will take much longer to extract ICG from the blood to the bile, as well as complete cellular washout. The longer interval may lower the false positive rate of tumor detection via NIRF imaging; however, there is no consensus yet on the optimal timing interval after ICG injection. And since most studies reported in HCC had ICG injected preoperatively as retention liver function test for hepatectomy, nearly all subjects had bilirubin level of less than $2 \mathrm{mg} / \mathrm{dL}$.

Fluorescence of the tumor identification reported is mainly relied on the late post-clearance effect that ICG stasis in the tumor while most dye has been washout by normal hepatocyte. The only exception was reported from China through intraoperative transarterial approach (28). Liu et al. developed the combination of the fluorescence goggle system and transarterial hepatic ICG delivery, and showed transarterial route of ICG facilitated rapid and selective uptake of ICG in HCC, providing higher imaging contrast between the tumors and normal hepatic tissue than the IV method. With this technique, identifying multifocal lesions and small tumor deposits was successfully achieved (28). However, the transarterial ICG injection to identify multifocal small tumor deposits may have the limitation that tumors enhanced only in a glimpse and the background parenchyma will bright-up and interfered the interpretation identifying well differentiated lesion. The NIRF utilities of segmentation mapping, tumor identification and cholangiography may interfere the interpretation among one another, as the fluorescence signal is based on the partial distribution of ICG by different timing. The latepost-clearance phase tumor identifying fluorescence can be obscure by the intraoperative IV ICG injection for identification of a portal vein territory for anatomical resection; while during fluorescence cholangiography, the bright parenchymal fluorescence is considered to be unnecessary enhancement that bring down the contrast between liver and bile duct, causing a high noise-to-signal ratio. van der Vorst et al. demonstrated the lowest noise-tosignal ratio can be reached when ICG was injected 72 hours prior to surgery (42). However, the subjects were based on colorectal liver metastasis, of which most patients were not cirrhotic.

Irrespective to the tumor florescence pattern, small subcapsular florescence nodules, which is unable to be seen on the white light view, can bright up under near infrared illumination. Criticism of its limitations to detect only superficial lesion, and also the necessity with intraoperative ultrasonography use to harvest a more accurate imaging have been emphasized (40). We applied the near infrared illumination during parenchymal transection, of which the potential risk of missed up daughter nodules can be easily seen on the rough cut surface distributed with clot 


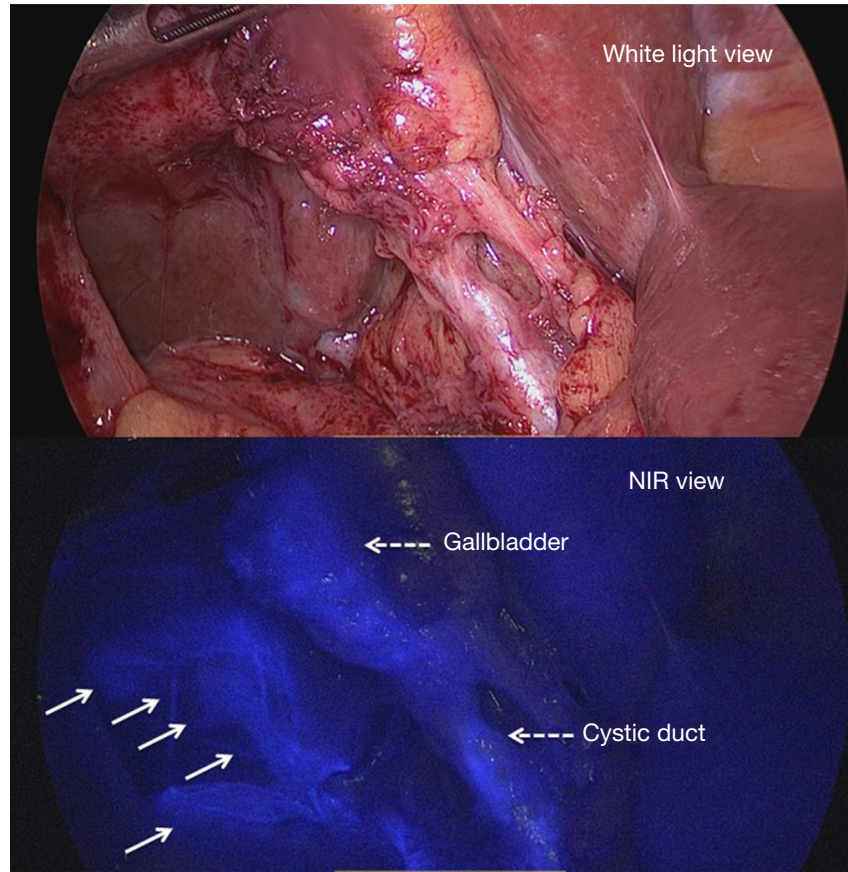

Figure 5 ICG contamination through lymphatic leakage. Once ICG leaks into the abdominal cavity, it binds with proteins and emits strong signals that occults critical structures. Intraoperative ICG spillage can cause fluorescent stain under NIR view that cannot be cleared immediately with suction or gauze mopping that can obscure our interpretation to the fluorescent signal. The arrows indicate lymphatic leak associated fluorescence contamination. ICG, indocyanine green; NIR, near-infrared.

and cautery burnt tissue (Figure 3,4). These mainly rely on the penetrating property of near infrared wave that can go through a certain depth of soft tissue.

Although Peyrat et al. (39) confirmed that ICG fluorescence imaging is a recommended tool for liver surgeon, as ICG fluorescence imaging can assist by identifying anatomical area, especially on altered liver. They mentioned the possibility of decreasing risk of liver relapse by detecting new metastases especially small and superficial lesions where US imaging is deficient. To date, it is inconclusive neither the new technology can be beneficial to short term outcomes in term of reduce blood loss or shorten operation time; nor long term results to prevent recurrence and to prolong cancer survival. Instead of conventional white light view, the additional fluorescence signal of potential lesions is a fact that more scientific explanations are needed in signal interpretation to further advance its advantage in clinical use. Yet pathologically,
Shibasaki et al. (11) demonstrated that the expression of organic anion transporting polypeptide 1B3 (OATP1B3), influx transporter, and multidrug resistance p-glycoprotein (MDR)-3, efflux transporter, were significantly higher in ICG-accumulated HCC (ICG-high HCC) than in ICGlow HCC. ICG was observed in the pseudo glands and bile canaliculi highly expressing MDR3. Furthermore, significantly lower disease-free and overall survival rates were found in patients with MDR3-negative HCC, in which the intratumoral accumulation of some phosphatidylcholine species was observed under imaging mass spectrometry. Thus, the intratumoral expression of MDR 3 affected the prognosis of HCC patients, possibly by changing the composition of cancer cell membranous phospholipids (11).

Another limitation of contaminated ICG spillage via lymphatic leakage (Figure 5), intraoperative portal vein or intraductal injection, can cause fluorescent stain under NIR view that cannot be cleared immediately with suction or gauze mopping that can obscure our interpretation to the fluorescent signal. Once ICG leaks into the abdominal cavity, it binds with proteins and emits strong signals that occults critical structures.

\section{Adverse reactions to the ICG injection}

The safety and continuity of the utility of NIR/ICG technology should be ensured by learning and education. Although the risk of adverse reactions to the ICG injection is very small (about $0.003 \%$ at doses exceeding $0.5 \mathrm{mg} / \mathrm{kg}$ ) (45), Marshall et al. reported a review of adverse events associated with intravenous ICG administration, death and anaphylaxis could occur with the trend of high dose or at the maximal dose of usage (46). ICG solutions aged after 7 and 30 days of daylight exposure containing only the degraded ICG could trigger severe cardiac arrhythmias within $10 \mathrm{sec}$ after being injected pigs and could result in death (47). To prevent adverse effect, one should always prepare the ICG solution right before injection. If additional injections are needed 6 hours after the first injection, a new vial of ICG should be used. Dosage below $0.5 \mathrm{mg} / \mathrm{kg}$ is recommended.

\section{Conclusions}

NIRF image-guidance during HCC surgery has the potential to improve patient management by visualizing tissue demarcation in real time, thereby increasing the completeness of surgery and decreasing the morbidity 
associated with damage to normal structures. Intraoperative imaging requires a synchronous interplay between contrast agents, tumor biology, imaging systems and image-analysis algorithms.

Given the interest in the field, the next decade should clarify the role of NIRF imaging in cancer surgery and the extent to which it empowers surgeons to improve patient outcomes.

\section{Acknowledgements}

We thank Hsing-Ju Wu, Research Assistant Center, Show Chwan Health Care System, Taiwan for editing the manuscript.

\section{Footnote}

Conflicts of Interest: The authors have no conflicts of interest to declare.

\section{References}

1. Makuuchi M, Kosuge T, Takayama T, et al. Surgery for small liver cancers. Semin Surg Oncol 1993;9:298-304.

2. Cherrick GR, Stein SW, Leevy CM, et al. Indocyanine green: observations on its physical properties, plasma decay, and hepatic extraction. J Clin Invest 1960;39:592-600.

3. Landsman ML, Kwant G, Mook GA, et al. Lightabsorbing properties, stability, and spectral stabilization of indocyanine green. J Appl Physiol 1976;40:575-83.

4. Aoki T, Yasuda D, Shimizu Y, et al. Image-guided liver mapping using fluorescence navigation system with indocyanine green for anatomical hepatic resection. World J Surg 2008;32:1763-7.

5. Spinoglio G, Priora F, Bianchi PP, et al. Real-time nearinfrared (NIR) fluorescent cholangiography in single-site robotic cholecystectomy (SSRC): a single-institutional prospective study. Surg Endosc 2013;27:2156-62.

6. Ishizawa T, Bandai Y, Kokudo N. Fluorescent cholangiography using indocyanine green for laparoscopic cholecystectomy: an initial experience. Arch Surg 2009;144:381-2.

7. Cheung TT, Han HS, She WH, et al. The Asia Pacific Consensus Statement on Laparoscopic Liver Resection for Hepatocellular Carcinoma: A Report from the 7th AsiaPacific Primary Liver Cancer Expert Meeting Held in Hong Kong. Liver Cancer 2018;7:28-39.

8. Satou S, Ishizawa T, Masuda K, et al. Indocyanine green fluorescent imaging for detecting extrahepatic metastasis of hepatocellular carcinoma. J Gastroenterol 2013;48:1136-43.

9. Kudo H, Ishizawa T, Tani K, et al. Visualization of subcapsular hepatic malignancy by indocyanine-green fluorescence imaging during laparoscopic hepatectomy. Surg Endosc 2014;28:2504-8.

10. Ishizawa T, Fukushima N, Shibahara J, et al. Real-time identification of liver cancers by using indocyanine green fluorescent imaging. Cancer 2009;115:2491-504.

11. Shibasaki Y, Sakaguchi T, Hiraide T, et al. Expression of indocyanine green-related transporters in hepatocellular carcinoma. J Surg Res 2015;193:567-76.

12. Zhang YM, Shi R, Hou JC, et al. Liver tumor boundaries identified intraoperatively using real-time indocyanine green fluorescence imaging. J Cancer Res Clin Oncol 2017;143:51-8.

13. Ishizawa T, Gumbs AA, Kokudo N, et al. Laparoscopic segmentectomy of the liver: from segment I to VIII. Ann Surg 2012;256:959-64.

14. Inoue Y, Arita J, Sakamoto T, et al. Anatomical Liver Resections Guided by 3-Dimensional Parenchymal Staining Using Fusion Indocyanine Green Fluorescence Imaging. Ann Surg 2015;262:105-11.

15. Mordon S, Devoisselle JM, Soulie-Begu S, et al. Indocyanine green: physicochemical factors affecting its fluorescence in vivo. Microvasc Res 1998;55:146-52.

16. Nairat M, Konar A, Kaniecki M, et al. Investigating the role of human serum albumin protein pocket on the excited state dynamics of indocyanine green using shaped femtosecond laser pulses. Phys Chem Chem Phys 2015;17:5872-7.

17. Yuan B, Chen N, Zhu Q. Emission and absorption properties of indocyanine green in Intralipid solution. J Biomed Opt 2004;9:497-503.

18. Gotoh K, Yamada T, Ishikawa O, et al. A novel imageguided surgery of hepatocellular carcinoma by indocyanine green fluorescence imaging navigation. J Surg Oncol 2009;100:75-9.

19. Sathirakul K, Suzuki H, Yasuda K, et al. Kinetic analysis of hepatobiliary transport of organic anions in Eisai hyperbilirubinemic mutant rats. J Pharmacol Exp Ther 1993;265:1301-12.

20. Ishizawa T, Masuda K, Urano Y, et al. Mechanistic background and clinical applications of indocyanine green fluorescence imaging of hepatocellular carcinoma. Ann Surg Oncol 2014;21:440-8.

21. Makuuchi M, Hasegawa H, Yamazaki S. Ultrasonically guided subsegmentectomy. Surg Gynecol Obstet 
1985;161:346-50.

22. Takasaki K. Glissonean pedicle transection method for hepatic resection: a new concept of liver segmentation. J Hepatobiliary Pancreat Surg 1998;5:286-91.

23. Sakoda M, Ueno S, Iino S, et al. Pure laparoscopic subsegmentectomy of the liver using a puncture method for the target portal branch under percutaneous ultrasound with artificial ascites. Surg Laparosc Endosc Percutan Tech 2013;23:e45-8.

24. Huang SW, Ou JJ, Wong HP. Laparoscopic left hepatectomy, anatomical resection with NIRF negative contrast. Asvide 2018;5:859. Available online: http://www. asvide.com/article/view/28308

25. Yamamoto S, Kim P, Kurokawa R, et al. Selective intraarterial injection of ICG for fluorescence angiography as a guide to extirpate perimedullary arteriovenous fistulas. Acta Neurochir (Wien) 2012;154:457-63.

26. Aoki T, Murakami M, Yasuda D, et al. Intraoperative fluorescent imaging using indocyanine green for liver mapping and cholangiography. J Hepatobiliary Pancreat Sci 2010;17:590-4.

27. Uchiyama K, Ueno M, Ozawa S, et al. Combined intraoperative use of contrast-enhanced ultrasonography imaging using a sonazoid and fluorescence navigation system with indocyanine green during anatomical hepatectomy. Langenbecks Arch Surg 2011;396:1101-7.

28. Liu Y, Zhao YM, Akers W, et al. First in-human intraoperative imaging of HCC using the fluorescence goggle system and transarterial delivery of near-infrared fluorescent imaging agent: a pilot study. Transl Res 2013;162:324-31.

29. Morita Y, Sakaguchi T, Unno N, et al. Detection of hepatocellular carcinomas with near-infrared fluorescence imaging using indocyanine green: its usefulness and limitation. Int J Clin Oncol 2013;18:232-41.

30. Sakoda M, Ueno S, Iino S, et al. Anatomical laparoscopic hepatectomy for hepatocellular carcinoma using indocyanine green fluorescence imaging. J Laparoendosc Adv Surg Tech A 2014;24:878-82.

31. Tanaka T, Takatsuki M, Hidaka M, et al. Is a fluorescence navigation system with indocyanine green effective enough to detect liver malignancies? J Hepatobiliary Pancreat Sci 2014;21:199-204.

32. Kitagawa N, Shinkai M, Mochizuki K, et al. Navigation using indocyanine green fluorescence imaging for hepatoblastoma pulmonary metastases surgery. Pediatr Surg Int 2015;31:407-11.

33. Miyata A, Ishizawa T, Tani K, et al. Reappraisal of a Dye-
Staining Technique for Anatomic Hepatectomy by the Concomitant Use of Indocyanine Green Fluorescence Imaging. J Am Coll Surg 2015;221:e27-36.

34. Kaibori M, Matsui K, Ishizaki M, et al. Intraoperative Detection of Superficial Liver Tumors by Fluorescence Imaging Using Indocyanine Green and 5-aminolevulinic Acid. Anticancer Res 2016;36:1841-9.

35. Terasawa M, Ishizawa T, Mise Y, et al. Applications of fusion-fluorescence imaging using indocyanine green in laparoscopic hepatectomy. Surg Endosc 2017;31:5111-8.

36. Boogerd LS, Handgraaf HJ, Lam HD, et al. Laparoscopic detection and resection of occult liver tumors of multiple cancer types using real-time near-infrared fluorescence guidance. Surg Endosc 2017;31:952-61.

37. Kobayashi Y, Kawaguchi Y, Kobayashi K, et al. Portal vein territory identification using indocyanine green fluorescence imaging: Technical details and short-term outcomes. J Surg Oncol 2017;116:921-31.

38. Lieto E, Galizia G, Cardella F, et al. Indocyanine Green Fluorescence Imaging-Guided Surgery in Primary and Metastatic Liver Tumors. Surg Innov 2018;25:62-8.

39. Peyrat P, Blanc E, Guillermet S, et al. HEPATOFLUO: A prospective monocentric study assessing the benefits of indocyanine green (ICG) fluorescence for hepatic surgery. J Surg Oncol 2018;117:922-7.

40. Lim C, Vibert E, Azoulay D, et al. Indocyanine green fluorescence imaging in the surgical management of liver cancers: current facts and future implications. J Visc Surg 2014;151:117-24.

41. Sheng QS, Lang R, He Q, et al. Indocyanine green clearance test and model for end-stage liver disease score of patients with liver cirrhosis. Hepatobiliary Pancreat Dis Int 2009;8:46-9.

42. van der Vorst JR, Schaafsma BE, Hutteman M, et al. Nearinfrared fluorescence-guided resection of colorectal liver metastases. Cancer 2013;119:3411-8.

43. Huang SW, Ou JJ, Wong HP. NIRF tumor identification in laparoscopic left sectionectomy, non-anatomical resection with NIRF illumination during parenchymal transection, of which the potential risk of missed up daughter nodules can be easily seen. Asvide 2018;5:860. Available online: http://www.asvide.com/article/ view/28309

44. Huang SW, Ou JJ, Wong HP. Although only superficial lesions could be detected, NIRF illumination of the cut surface provided additional information to reconfirm the cut margin was free of gross residual tumor, even on the rough cut surface distributed with clot and cautery burnt 
tissue. Asvide 2018;5:861. Available online: http://www. asvide.com/article/view/28310

45. Speich R, Saesseli B, Hoffmann U, et al. Anaphylactoid reactions after indocyanine-green administration. Ann Intern Med 1988;109:345-6.

46. Marshall MV, Rasmussen JC, Tan IC, et al. Near-Infrared

doi: $10.21037 / \operatorname{tgh} .2018 .10 .15$

Cite this article as: Huang SW, Ou JJ, Wong HP. The use of indocyanine green imaging technique in patient with hepatocellular carcinoma. Transl Gastroenterol Hepatol 2018;3:95.
Fluorescence Imaging in Humans with Indocyanine Green: A Review and Update. Open Surg Oncol J 2010;2:12-25.

47. Ott P, Keiding S, Johnsen AH, et al. Hepatic removal of two fractions of indocyanine green after bolus injection in anesthetized pigs. Am J Physiol 1994;266:G1108-22. 